\title{
Partner Tiering in Display Advertising
}

\author{
Anand Bhalgat ${ }^{*}$ \\ Facebook Inc \\ bhalgat@fb.com
}

\author{
Nitish Korula \\ Google Research \\ nitish@google.com
}

\author{
Max Lin \\ Google Inc \\ whilin@google.com
}

\author{
Vahab Mirrokni \\ Google Research \\ mirrokni@google.com
}

\begin{abstract}
Display ads on the Internet are often sold by publishers to advertisers in bundles of thousands or millions of impressions over a particular time period. The ad delivery systems assign ads to pages on behalf of publishers to satisfy these contracts, and at the same time, try to maximize the overall quality of assignment. This is usually modeled in the literature as an online allocation problem, where contracts are represented by overall delivery constraints.

However an important aspect of these contracts is missed by the classical formulation: a majority of these contracts are not between advertisers and publishers; a set of publishers is typically represented by a middle-man and advertisers buy inventory from the middle man. As publishers vary in quality and importance, advertisers prefer these publishers differently. Similarly, as the inventory of ads is limited, ad-delivery engine needs to prefer a high-quality publisher over a low quality publisher for supplying ads. We formulate this problem as a hierarchical online matching problem where each incoming impression has a level indicating its importance, and study its theoretical properties. We also design practical solutions to this problem and study their performance on real data sets.
\end{abstract}

\section{Categories and Subject Descriptors}

F.2.m [Theory of Computation]: Analysis of Algorithms and Problem Complexity-Miscellaneous; H.4.m [Information Systems Applications]: Miscellaneous

\section{Keywords}

Display Ads Allocation; Partner-Tiering; Online Matching

\section{INTRODUCTION}

Worldwide online advertising revenues are estimated to exceed US $\$ 118$ billion in 2013 , with recent annual growth

\footnotetext{
*Work done while interning at Google NYC.
}

Permission to make digital or hard copies of part or all of this work for personal or classroom use is granted without fee provided that copies are not made or distributed for profit or commercial advantage, and that copies bear this notice and the full citation on the first page. Copyrights for third-party components of this work must be honored. For all other uses, contact the owner/author(s). Copyright is held by the author/owner(s).

WSDM'14, February 24-28, 2014, New York, New York, USA.

ACM 978-1-4503-2351-2/14/02.

http://dx.doi.org/10.1145/2556195.2556258. rates of $15 \%$ [1]. Motivated by practical problems in search and display advertising, recent years have seen the development of a rich theory of online allocation problems and algorithms. As the online advertising industry has evolved, research focus has shifted from search advertising $[15,10,8]$ to contract-based display advertising $[12,13,11,6,7]$ and auction-based advertising exchanges $[4,14,5]$. In the study of many of these models, an implicit assumption has been a relative scarcity of advertising opportunities (the supply of user-generated impressions), compared to a relative surplus of demand by advertisers. When this is not the case, for example, the standard budgeted allocation model $[15,10,8]$ representing search advertising is not interesting: If there were a significant surplus of user queries / advertising opportunities, all advertisers will eventually spend their entire budgets, regardless of the allocation algorithm used. In this paper, we consider a problem motivated by an increasing availability of online advertising inventory in several applications, including in-app mobile advertising, web-based display advertising, and also video advertising for TV content creators. In the following, we elaborate on these motivating applications in more details.

We begin with the in-app mobile advertising. Given an unprecedented increase in popularity of smart-phones in recent past and ever-growing amount of time spent on them (according to mobile apps analytic firm Flurry, US consumers now spend over two and a half hours per day on mobile devices, almost the same as time spent watching television [2]), the in-app advertisers have an increasingly large available inventory to which they can target their ads. However, with the large number of apps that are advertsing inventory sources, (the Google Play store lists over a million apps, and Apple's iTunes App Store offers a similar number), it is clearly infeasible for advertisers to reach individual agreements with the creators of each app. This gives rise to the existence of platforms such as Velti or Millennial Media, which act as middlemen. These platforms partner with a number of advertising inventory sources; in this case, app creators. In the rest of the paper, we refer to an individual advertising inventory source in the platform as the partner.

Advertisers can approach the platform and create a single advertising campaign with a unified budget and other properties, but have their ads appear on the apps of many different partners. This nature of contracts introduces new challenges: From the perspective of an advertiser, not all inventory supplied by the platform is equally good; advertisers often value premium inventory (corresponding to users of apps such as the New York Times or the Economist) much 
more than other inventory sources (such as users of some gaming apps). However, it may not be possible for advertisers to explicitly list their preferences among all the different inventory sources, or to accurately estimate what fraction of inventory will come from different sources. In this situation, advertisers must rely on the platform (which implicitly or explicitly ranks inventory sources) to give them an appropriate share of premium inventory. From the platform's perspective, the advertising demand is constrained; and it makes business sense to match ads to premium partners over lower quality partners:

(a) If the platform satisfies as much demand as possible with inventory from premium partners, then the advertisers are more likely to return to the platform.

(b) If the fill rate for a premium partner (the fraction of impressions for a partner to which the platform assign an ad) is higher, then the premium partner sees more revenue and is more likely to stick with the platform. It is important for a platform to keep the premium partners in its inventory, as this helps attract and retain advertisers.

A similar problem occurs in web-based display advertising. In the past, advertisers made contracts with individual publishers such as the Wall Street Journal, reserving a certain number of impressions, and an ad serving platform (e.g. OpenX, DoubleClick, 24/7 Real Media) assigns ads to publisher's impressions at run-time. However, it is increasingly difficult for advertisers to reach agreements with more than a few top publishers, leading to the rise of various online exchanges (such as Google AdExchange or Yahoo! Right Media) that act as central clearinghouses or platforms where publishers can sell their remaining unallocated inventory. It is important to note, the ads served under advertiserpublisher reservation contracts are premium ads, commanding a significantly higher CPM than the price a publisher gets for his leftover inventory on the exchanges.

In this new business model of programmatic contracts, advertisers want to create a single unified campaign with the platform, which decides how to satisfy this advertising demand from different (contract-based as well as exchangebased) partner sources. One natural objective for the platform is to satisfy the demand in a way which maximizes the advertiser value (typically measured in number of clicks or conversions). In addition, the platform may also wish to ensure a high fill rate for higher-priority or premium partners, because this directly affects platform revenue and gives premium partners more incentive to stay with the platform.

Finally, a similar problem is faced by content creators, such as CBS, and this content is viewed by consumers on websites, such as YouTube, that serves as distribution partners. Here, the content creator plays the role of the middleman, making contracts with advertisers to show ads when their content is played on any partner website. Each distribution partner may have a different business relationship and revenue sharing arrangement with the creator, and so the ad serving platform should prefer to display ads on higher-value partners to generate more revenue for the content creator. Products such as MRM from FreeWheel solve this problem for content creators.

\subsection{Our Contributions}

Our first contribution is to provide a formal model of the common problem faced in all the applications above, and provide a benchmark to compare the performance of algo-

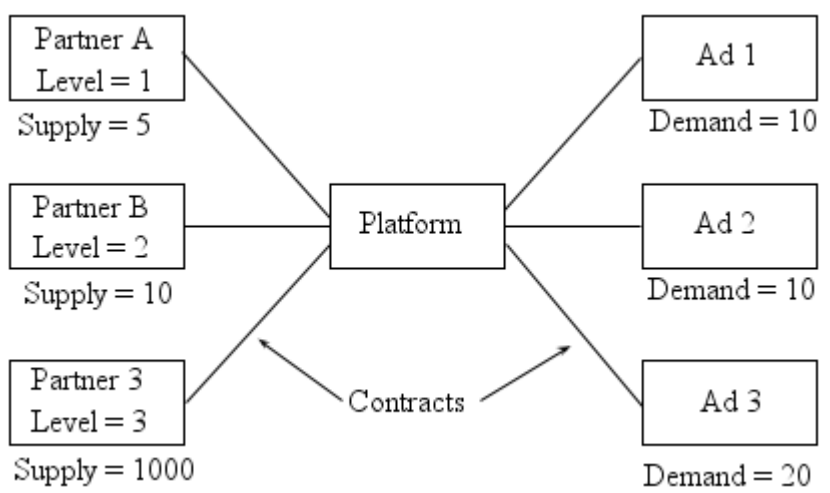

Figure 1: Three partners at different levels represented by a single platform. Supply at level 1 and 2 is constrained, however the overall supply exceeds the demand.

rithms for this setting. Second, we design algorithms for both the offline and online settings and prove guarantees on their performance (with respect to the benchmark matching) for some cases of the problem. In particular, we observe that the exact optimum solution can be computed in polynomialtime for unweighted graphs, and present a constant-factor approximation algorithm for weighted graphs. Moreover, we show that one of our efficient heuristic online algorithms provide an improved approximation algorithm for the problem in the online stochastic arrival model. Finally, we study several efficient heuristics (DUAL-SCALE and EW-SCALE) for the problem and analyze their performance on real data-sets from a leading online advertising corporation. Our empirical study shows that the DUAL-SCALE algorithm performs better compared to the EW-SCALE algorithm.

We next discuss these aspects in detail; we begin with describing the overall model.

The Model: There are multiple partners that are represented by a common platform. Each partner is associated with a priority level reflecting the general value of its inventory. Advertisers demand inventory from the platform that can be satisfied by partners at different levels. In general, there is a surplus of inventory from the partners, however the supply of premium inventory (high priority levels) is constrained compared to advertisers' demands. See Fig. 1 for an illustration.

Platform Objectives: Next we discuss the multiple objectives of the platform: First, it must satisfy the demand of the advertisers. Second, it should ensure a higher fill-rate for higher-level or premium partners: This may be to increase platform revenue, advertiser happiness, or long-term platform benefits by ensuring the continued association of premium partners. Third, in some applications, inventory may have different values for different advertisers (based on click probabilities, for instance) independent of the level of its source; in these settings, an additional objective is to maximize the total value of the allocated impressions.

Note that given the surplus of inventory, we do not expect that all (or even a large fraction of) inventory will be assigned to advertisers. Thus, the second objective is important: the platform needs to ensure that the premium inventory is not left unassigned. Often, these platform goals are contradictory and it is not clear which assignment is optimal; 

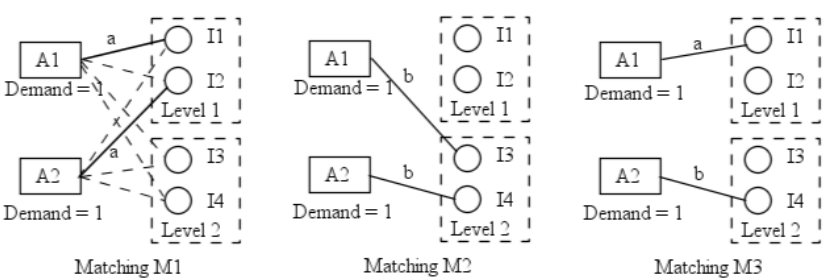

Figure 2: The set of possible matchings in Example 1.1

we illustrate with an example, where different matchings are optimal in this case based on different platform objectives:

Example 1.1 There are two advertisers $\mathcal{A}_{1}$ and $\mathcal{A}_{2}$. Both advertisers have demand 1 . There are two partners: $P_{1}$ and $P_{2}$, at priority levels 1 and 2 respectively. Each partner supplies two impressions; the weight (CTR) of each impression from partner 1 for both advertisers is a and weight (CTR) of each impression from partner 2 is $b$ for both advertisers; further we have $a<b$. The example and some of possible matchings in this case are illustrated in Fig. 1.1.

In this example,

- The matching $M_{1}$ is optimal when the platform wants to maximize the fill rate for the premium inventory; however it is sub-optimal in terms of total number of clicks generated by the platform for its advertisers.

- The matching $M_{2}$ maximizes the total number of clicks; however the fill rate achieved for the premium impressions is nil.

- The matching $M_{3}$ does not achieve the maximum possible fill rate for premium impressions, and it does not generate maximum number of clicks. However, it is more fair to both objectives compared to $\mathcal{M}_{1}$ and $\mathcal{M}_{2}$.

Thus different platform goals lead to different optimal matchings. Even if one were to assume that the platform's final objective can be represented as a utility function over these contradictory goals, such function can be arbitrarily complex; and it is even unrealistic to expect that the utility function is known entirely (even to the platform) or can be completely expressed. Typically, the platform may be able to give his choice between a pair of matchings.

Benchmark: Given the complexities in defining the platform's utility function, we take a worst case approach and compare the matching against a set of $L$ distinct matchings ( $L$ : \#(impression levels)) that serves as a benchmark. The $\ell$ th matching in this set is an optimal off-line matching, considering impressions only in levels 1 through $\ell$; we indicate this matching (as well as its weight) by $\mathrm{OPT}_{\ell}$. Given any matching $\mathcal{M}$, we denote the weight of $\mathcal{M}$ considering impressions in first $\ell$ levels by $W_{i}(\mathcal{M})$, and let $\alpha_{\ell}(\mathcal{M})=\frac{W_{\ell}(\mathcal{M})}{\mathrm{OPT}_{\ell}}$. We represent the performance of $\mathcal{M}$ by its approximation factor $\left(\alpha_{1}(\mathcal{M}), \alpha_{2}(\mathcal{M}), \ldots, \alpha_{L}(\mathcal{M})\right)$. Thus the benchmark serves as an indicator for the quality of a matching in absence of quantitative importance of impressions at various levels, which as discussed earlier in the section, may not exist.

\section{Previous Work.}

Our work is related to online ad allocation problems, including the Display Ads Allocation (DA) problem [12, 11, 3, $17,6]$, and the $A d W o r d s ~(A W)$ problem $[15,10]$. In both of these problems, the platform must assign online impressions to an inventory of ads, optimizing efficiency or revenue of the allocation while respecting pre-specified contracts. Both of these problems have been studied in the competitive adversarial model $[15,12,8]$ and the stochastic random-arrival model $[10,11,3,17,16]$. We briefly summarize these two models. In a DA problem, advertiser $\mathcal{A}_{i}$ has capacity of $N(i)$ impressions; the platform allocates impressions to advertisers in order to maximize the total weight of matching (i.e. number of clicks) subject to each advertiser's capacity constraint. Whereas, in the AW problem, $\mathcal{A}_{i}$ has a budget $B(i)$ on the total spend and assigning impression $j$ to $\mathcal{A}_{i}$ consumes $w(i, j)$ units of $\mathcal{A}_{i}$ 's budget.

Both the DA problem (because of the standard free-disposal assumption) and the AW problem can be seen as supplyconstrained, i.e., the complexity of the problem is based on a limited supply of impressions with the platform compared to the advertiser demand. To illustrate this more clearly, recall that in the case of the AW problem, if there is a significant surplus of user queries / advertising opportunities, all advertisers will eventually spend their entire budgets, regardless of the allocation algorithm used. In comparison, the partner-tiering problem is demand-constrained: the platform has a large supply of impressions, but the advertising demand is limited, hence the platform must intelligently ensure a higher fill-rate for higher-priority inventory.

Organization: We formally define the model and provide important definitions in 2. Then we describe our results for offline and online setting in Sections 3 and 4 respectively. Finally, we highlight important empirical observations in Section 5.

\section{PRELIMINARIES}

We already discussed our basic model in Section 1.1. Here we describe the notation behind the abstract model that we will use throughout the paper. There is a set of advertisers $\mathcal{A}_{1}, \mathcal{A}_{2}, \ldots, \mathcal{A}_{n}$. Advertiser $\mathcal{A}_{i}$ wants $N(i)$ impressions. There are $m$ partners $P_{1}, P_{2}, \ldots, P_{m}$ represented by a platform (a middleman). Each partner (specifically, the set of impressions from a partner) is associated with a priority level between 1 and $L$. We denote $P_{i}$ 's priority level by $\mathcal{L}\left(P_{i}\right)$.

In an online-arrival setting, impressions arrive online oneby-one; we denote the $j^{\text {th }}$ impression in the arrival sequence by $\mathcal{I}_{j}$. Each impression belongs to one partner and it inherits the partner's priority level. We denote $\mathcal{I}_{j}$ 's priority level by $\mathcal{L}(j)$. When impression $\mathcal{I}_{j}$ arrives, the set of advertisers interested in it is revealed. We denote the weight(CTR) of assignment between $\mathcal{A}_{i}$ and $\mathcal{I}_{j}$ by $w(i, j)$.

We mainly consider the problem in the online arrival model, but in order to understand the underlying optimization problem, and for completeness, we also consider an offline variant of the problem: in an off-line setting, all impressions and their edge weights are known upfront to the algorithm.

In this paper, we consider un-weighted as well as weighted settings: in the un-weighted setting, $w(i, j) \in\{0,1\}$, where 0 indicates the absence of an edge and 1 indicates the presence of an edge. The weighted setting has no such restriction and arbitrary non-negative edge weights are allowed. 


\section{ALGORITHMS IN OFF-LINE SETTING}

In this section, we develop algorithms to compute (approximately) optimal solutions to partner-tiering in the offline setting. As discussed earlier, even though the online settings is the focus of this paper, understanding the complexity and approximability of the problem in offline setting is important in understanding the problem in the online setting. We establish our result for the un-weighted case in Theorem 1, and follow it with our results for the weighted case (Theorem 2).

Theorem 1 For unweighted graphs in the off-line setting, there exists a matching that is an optimal matching, i.e., $(1,1, \ldots, 1)$-approximation. Further, there is a polynomialtime algorithm to compute such an optimal matching.

Proof. We give a proof sketch: Let $m$ be the total number of impressions in the sequence. We assign a weight to each edge in the graph as follows: the weight of an edge between an impression at level $\ell$ and an (interested) advertiser is $1+\frac{L-1-\ell}{m L}$. It can be easily shown that the maximum-weight matching in this graph is a $(1,1, \ldots, 1)$ approximation.

\section{Weighted Graphs.}

Next, we address the question of computing the optimal matching for a weighted graph in the offline setting. We first establish shortcomings of the standard free disposal assumption; we follow it with a relaxed definition for free disposal and establish theoretical results under this assumption.

Free Disposal Assumption: The DisplayAds problem is known to be inapproximable (to compute the maximum weight matching) without the free disposal assumption [12]. This assumption states that giving an advertiser more impressions than his demand does not decrease his value. In other words, advertiser $\mathcal{A}_{i}$ can be assigned more impressions than his demand $N(i)$; when we consider his value in the weight of the matching, we count the $N(i)$ impressions of highest weight and (freely) dispose of the remaining impressions.

We establish in the next example, that the standard free disposal assumption fails to capture the complexities when there are multiple layers of impressions and the partnertiering problem remains inapproximable. We then introduce a tier-based free disposal assumption; and establish useful results under this assumption.

Example 3.1 There is one advertiser $\mathcal{A}_{i}$ with demand $N(i)$. There are $L$ levels of impressions. Each level $\ell$ has $N(i)$ impressions; the weight of an impression in level $\ell$ is $k^{\ell}$ for some large constant $k>1$. All impressions are in $\mathcal{A}_{i}$ 's target set. Given any assignment of impressions to $\mathcal{A}_{i}$; it can be easily seen that no fixed set of $N(i)$ impressions is simultaneously competitive against $\mathrm{OPT}_{1}$ through $\mathrm{OPT}_{\ell}$.

Tier-based Free Disposal Assumption: Under this assumption, we are allowed to over-deliver impressions, however we do not need to fix the set of impressions to be disposed of when an advertiser is over-delivered. While computing the performance for advertiser $\mathcal{A}_{i}$ from the first $\ell$ levels, we can choose the $N(i)$ highest-weight impressions in the first $\ell$ levels. Thus, the set of impressions that count towards the objective can be a function of the level.
We illustrate the implications of this assumption in case of Example 3.1: here, we assign $N(i)$ impressions from each level to $\mathcal{A}_{i}$. When we measure the quality of this matching for first $\ell$ levels, the best $N(i)$ impressions in first $\ell$ levels are $N(i)$ impression from level $\ell$ each of weight $k^{\ell}$. Thus, the assignment is a $(1,1,1, \ldots, 1)$-approximate matching.

We next give an algorithm to compute an approximately optimal matching in this setting (Theorem 2), and show existence of instances which do not permit $(1,1, \ldots, 1)$-approximate matchings (Example 3.2). We leave the question of computing tight upper bounds in this case as future work.

Theorem 2 For weighted graphs in the offline setting, there exists a matching that is a $(1-1 / e, 1-1 / e, \ldots, 1-1 / e)$ approximation, under the tier-based free-disposal assumption.

Proof. We highlight the main ideas of the proof and defer the complete proof to the full version of the paper. We order impressions according to their priority levels, where impressions in level $\ell$ appear before impressions in level $\ell+1$, for each $\ell$. We then solve the DisplayAds instance assuming this order of impression arrival using the PD-Exp algorithm by Feldmen et al [12], neglecting impression levels. It can be shown that the matching computed in this process is a $(1-1 / e, 1-1 / e, \ldots, 1-1 / e)$-approximate matching under the tier-based free disposal assumption. The crucial idea in the proof is that under the new free disposal assumption, the matching computed for a prefix of a sequence does not loose its value by subsequent insertion of impressions.

Example 3.2 There are two levels of impressions and two advertisers, each with demand $N$. There are $N$ impressions in each level. Impressions in the first level have edge weights of 0.1 and 0.2 for $\mathcal{A}_{1}$ and $\mathcal{A}_{2}$ respectively; and the edge weights for the second level impressions are 0.3 and 0.4 respectively. Clearly, the optimal matching considering only the first-level impressions assigns all impressions to $\mathcal{A}_{2}$; whereas the optimal matching considering both levels assigns the first-level impressions to $\mathcal{A}_{1}$ and the second-level impressions to $\mathcal{A}_{2}$. Further, it can be easily seen that no matching is a $(1,1)$-approximate matching.

\section{ALGORITHMS IN ONLINE SETTING}

In this section, we develop practical algorithms for partnertiering when impressions arrive online, and establish useful theoretical guarantees in online adversarial and online stochastic models. These algorithms are inspired by the dual-based techniques developed in past for online matching (in absence of impressions' levels); see e.g. [12, 6]. We begin with the dual scaling (DUAL-SCALE) algorithm.

\subsection{Dual Scaling}

In this algorithm, we are given a discount factor $\beta \in[0,1]$ that is used to scale the value of dual in a dual-based algorithm, depending upon the level of an incoming impression and the prior assignment to an advertiser. We illustrate the details: let weight $(i, \ell)$ be the total weight of impressions in level $j$ assigned to advertiser $\mathcal{A}_{i}$ so far. For an incoming impression at level $\ell$, his scaled dual $\gamma(i, \ell)$ (for level $\ell$ ) is defined as

$$
\gamma(i, \ell)=\frac{\sum_{l \leq \ell} \text { weight }(i, l)+\beta \times\left(\sum_{l>\ell} \text { weight }(i, l)\right)}{N(i)}
$$


Impression Assignment: The incoming impression $\mathcal{I}_{j}$ at level $\ell$ is assigned to an advertiser that maximizes the marginal with respect to the scaled dual, i.e.

$$
\operatorname{argmax}_{i}\{w(i, j)-\gamma(i, \ell)\}
$$

The $\beta$ serves as a knob that indicates the importance of higher level impressions over the overall delivery quality. Its value can be chosen statically; or one can also use onlineadaptive techniques (such as PID-control).

Implementation details for computing dual variables: For each advertiser $\mathcal{A}_{i}$, we need to store up to top $N(i)$ impressions at each level. When $N(i)$ or the number of levels is large, the overhead can be reduced by storing the distribution over edge-weights at each level for an advertiser (at a small loss in accuracy).

The two extreme cases of this algorithm are:

When $\beta=0$, the dual variable for $\mathcal{A}_{i}$ at level $\ell$ completely ignores impressions assigned to him in levels $\ell+1$ and lower.

When $\beta=1$, all impressions assigned to an advertiser are considered identical while computing his dual particular to a level. In this case, the algorithm degenerates to the PD-Avg algorithm from [12].

We next establish useful theoretical guarantees of the DUALSCALE algorithm for un-weighted graphs at these two extremes in an online arrival model.

\section{Un-weighted Graphs: $\beta=0$.}

We establish theoretical guarantees on the performance of the DUAL-SCALE algorithm for $\beta=0$ in adversarial as well as stochastic arrival model. Interstingly, the approximation ratio of the algorithm is very different for these two arrival models: in the adversarial arrival model, its performance drop to $o(1)$ as the number of levels increases (Example 4.1); whereas it performs very well in a stochastic arrival model (Theorem 3). Further, in contrast to its guarantees in the adversarial arrival model, we observe that the DUAL-SCALE algorithm performs very well on real data sets for small values of $\beta$ (see Section 5).

Example 4.1 Consider an instance with $n+\sqrt{n}$ advertisers. Each advertiser has demand $t$. There are $n$ levels of impressions, each level has $t$ impressions, and the impressions in level $i$ are eligible for advertisers $i$ and $n+1$ through $n+\sqrt{n}$. Clearly the optimal matching considering impressions in all levels has weight nt whereas the matching generated by the DUAL-SCALE algorithm has weight $2 \sqrt{n} t$.

Stochastic Arrival Model: We next define the stochastic arrival model considered in our study. We are given a set of impression-types $\mathcal{T}_{1}, \mathcal{T}_{2}, \ldots, \mathcal{T}_{m}$. Each incoming impression is infinitesimally small and belongs to one of the types. All impressions belonging to the same type are identical for an advertiser, and we use $\mathcal{N}\left(\mathcal{T}_{j}\right)$ to indicate the set of advertisers that are interested in impressions of type $\mathcal{T}_{j}$. Each impression type is associated with a non-zero arrival rate (per unit time), represented as the fraction of all incoming impressions in a unit time. The problem of online matching has been extensively studied in this model; see e.g. [9]. We note, this arrival model is different than the randompermutation-arrival model studied in past [10, 11, 3, 17].
We further assume that all impressions of a given type come from the same partner and hence have the same priority level. Next, (Theorem 3), we establish the approximation guarantee of the DUAL-SCALE algorithm for $\beta=0$; we then show the tightness of this guarantee in Example 4.2.

Theorem 3 For un-weighted graphs and the stochastic arrival model, the approximation ratio of the DUAL-SCALE algorithm for $\beta=0$ is $(1,3 / 4,13 / 18, \ldots, 1-1 / e)$.

Proof. Due to space constraints, we briefly outline the central ideas and defer the complete proof to the full version of the paper.

As each impression-type is associated with a per unit-time arrival rate, we associate temporal aspect with impressions' arrival. We use 0 and $t_{\text {end }}$ to indicate the start and the end time of the impressions' arrival. Further, for any intermediate time $t$, we use $t_{+}$and $t_{-}$to indicate the time $\lim _{t^{\prime} \rightarrow t_{+}} t^{\prime}$ and $\lim _{t^{\prime} \rightarrow t_{-}} t^{\prime}$ respectively.

Given any impression type $\mathcal{T}_{j}$, let $\delta(i, j, t)$ be the rate of assignment of $\mathcal{T}_{j}$ to advertiser $\mathcal{A}_{i}$ at time $t$, let $\gamma(i, \ell, t)$ be the value of $\gamma(i, \ell)$ at time $t$. We next state important invariants of the algorithm; its proof follows by induction on $t$ and $\ell$.

Lemma 4.1 Given an arbitrary impression type $\mathcal{T}_{j}$, let $\ell$ be its priority level, then for each advertiser $\mathcal{A}_{i} \in \mathcal{N}\left(\mathcal{T}_{j}\right)$ :

- For each $t>0$, either (a) $\delta(i, j, t)=\delta\left(i, j, t_{-}\right)$and $\gamma(i, \ell, t)<1$ or $(b) \delta(i, j, t)=0$ and $\gamma(i, \ell, t)=1$.

- If $\delta\left(i_{1}, j, 0_{+}\right) \neq 0$, and $\delta\left(i_{2}, j, 0_{+}\right) \neq 0$, then $\gamma\left(i_{1}, \ell, t\right)=$ $\gamma\left(i_{2}, \ell, t\right)$ for each $t>0$.

- If $\mathcal{A}_{i_{1}}, \mathcal{A}_{i_{2}} \in \mathcal{N}\left(\mathcal{T}_{j}\right)$ and $\delta\left(i_{1}, j, 0_{+}\right)>0, \delta\left(i_{2}, j, 0_{+}\right)=$ 0 , then for each $t>0, \gamma\left(i_{1}, l, t\right) \leq \gamma\left(i_{2}, l, t\right)$

For the purpose of analysis of the approximation factor, we consider a bipartite graph, with advertisers on one side and impression types on the another side. The edges in the graph are from advertisers to impressions-types in their target set. The vertices in the graph have weights, indicating their demands (in case of advertisers) or supply (in case of impression types). Further, we consider a (weighted) matching induced on this graph by the impressions' assignment up to time $t_{\text {end }}$; the weight on each edge in this matching is the volume of assignment between a given advertiser-impression type pair. We now prove our claim for the first level.

Lemma 4.2 The matching computed by the DUAL-SCALE algorithm for $\beta=0$ is optimal for the first level.

Proof. We note, impressions at level $\geq 2$ can be completely ignored for the purpose of this proof.

Consider any impression type $\mathcal{T}_{j}$ belonging to level 1 such that $\exists \mathcal{A}_{i} \in \mathcal{N}\left(\mathcal{T}_{j}\right)$ with $\delta\left(i, j, 0_{+}\right)>0$ and $\gamma(i, 1, t)=1$ at some $t<t_{\text {end }}$ (i.e. impressions from $\mathcal{T}_{i}$ are assigned to at least one advertiser whose complete demand is met before the end of the algorithm). It suffices to show that no augmenting paths beginning at $\mathcal{T}_{j}$ exists in the matching computed by the DUAL-SCALE algorithm. Using Lemma 4.1, for each $\mathcal{A}_{k} \in \mathcal{N}\left(\mathcal{T}_{j}\right)$, we have $\gamma(k, 1, t)=1$. Further, applying this argument recursively, we get that any alternating path beginning in $\mathcal{T}_{j}$ does not end at $\mathcal{A}_{l}$ with $\gamma\left(l, 1, t_{\text {end }}\right)<0$, implying that there are no augmenting paths in the matching computed by the algorithm. This establishes the lemma. 
The proof for higher levels is more involved and is deferred to the full version of the paper. We explain the main ideas behind the proof using level 2 as an example: for level 2 impressions, the matching graph at the end of the algorithm does not have any augmenting paths that use only one level (either level 1 or 2) of impressions. However, such path may begin with a level 2 impression and end with an advertiser whose demand is not completely met, and this path will have intermediate level 1 impressions (and no level 2 impressions). The main challenge is to bound the total volume of these paths. Towards this purpose, we use the following key property: when we trace any potential augmenting path and consider a sequence of advertisers on this path in the same order, then a non-decreasing fraction of advertiser's demand is met with level 1 impressions. Hence, the terminating advertiser on this path always has a greater fraction of his demand met through level 1 impressions than the first (and hence over-allocated) advertiser on this path. We then bound the volume of these augmenting paths by establishing important structural properties.

Example 4.2 Consider an instance with $n$ advertisers and $n$ impression types; $t \rightarrow \infty$ impressions of each type arrive and the capacity for each advertiser is $t$. Impressions of type $\mathcal{T}_{1}$ are eligible for all advertisers, $\mathcal{T}_{2}$ for first $n-1$ advertisers, $\mathcal{T}_{3}$ for first $n-2$ advertisers, and so on. Further, impressions of type $\mathcal{T}_{j}$ belong to level $j$ for each $j$. The DUAL-SCALE algorithm divides the impressions of each type equally among eligible advertisers. Thus, the weight of the matching achieved by the DUAL-SCALE algorithm is exactly $(t, 6 t / 4,33 t / 18, \ldots, n t(1-1 / e))$; whereas the weight of the optimal matching is $(t, 2 t, 3 t, \ldots, n t)$.

\section{Un-weighted Graphs for $\beta=1$.}

For un-weighted graphs, the dual-scaling algorithm for $\beta=1$ assigns an incoming impression to the advertiser with maximum unsatisfied demand (as a fraction of his total demand). Thus the impression remains unassigned when all interested advertisers have met their entire demand. We show that a slight modification to this algorithm has an interesting theoretical guarantee.

We refine the assignment of the algorithm when all eligible advertisers have completely used their demand: in this case, for an incoming impression $\mathcal{I}_{j}$ at level $\ell$, let $k \in[\ell, L]$ be the largest integer such that, there exists an advertiser $\mathcal{A}_{i}$ who has been assigned less than $N(i)$ impressions in first $k$ levels. Then, we assign the impression to $\mathcal{A}_{i}$. If no such $k$ exists, then the impression is left unassigned.

We note two invariants maintained by the algorithm; as a corollary, we get Lemma 4.3.

- For each level $\ell<L$, and for each prefix of $j$ incoming impressions; the matching generated by the algorithm is maximal, considering the impressions in first $\ell$ levels.

- For level $L$ and for each prefix of $j$ incoming impressions, the matching generated by the algorithm is (11/e)-approximate: this follows as the algorithm always assigns according to the dual based algorithm that treats all levels equally.

Lemma 4.3 For $\beta=1$, the dual scaling algorithm is a $(1 / 2,1 / 2, \ldots, 1 / 2,1-1 / e)$-approximation.

\subsection{Edge-Weight Scaling}

This is an additional heuristic that we consider in our empirical study. In the edge-weight scaling (EW-SCALING) algorithm, we are given scaling factors $\mathrm{sc}_{1} \geq \mathrm{sc}_{2} \geq \ldots \mathrm{sc}_{L}$ : when an impression at level $\ell$ arrives, its weight is scaled by a factor $\mathbf{s c}_{\ell}$. We define the dual variable $\gamma(i)$ for advertiser $\mathcal{A}_{i}$ as the average scaled weight of impressions assigned to him. The incoming impression $\mathcal{I}_{j}$ at level $\ell$ is assigned to the advertiser that maximizes:

$$
\operatorname{argmax}_{i}\left(\mathbf{s c}_{\ell} \times w(i, j)-\gamma(i)\right)
$$

Different scaling factors can be used based on the delivery constraints and relative importance of the higher level impressions. When scaling factors are all 1, it is equivalent to treating all levels equally.

\section{EXPERIMENTAL EVALUATION}

Dataset: We study the performance of our algorithms on display ads data for a set of 3 anonymous 'publishers' (each representing multiple partners) working with a leading display ads serving corporation. We collect the entire set of impressions for a period of one week for each publisher. The number of impressions in a data set ranges from $200 K$ to $3 M$. For each incoming impression, the information about the set of interested advertisers, their (predicted) CTR values and the impression type is available. Further, the delivery contracts for each advertiser are also available.

The impressions do not reveal the partner-source (and hence their level); however we have information about the impression type (which represents the advertisers' targeting criteria for the impression). Hence, we generate synthetic values of priority levels for impressions based on their types. We associate each impression a priority level between 1 and 10 using three different techniques, giving us three types of data sets for each publisher.

- Method A: We statically associate each impression type with a priority level. When an impression arrives, this information is used to generate its priority level.

- Method B: For each incoming impression, we associate it a priority level based on its type with $80 \%$ probability and choose a uniformly random priority level between 1 and 10 otherwise.

- Method C: Each incoming impression is given a uniformly random priority level between 1 and 10 .

We note, these three data sets represent three different types of workloads: The first data set represents a workload with little overlap in the impressions' types across partners, and hence an advertiser's target set of impressions is concentrated at one (or few) level(s); the second data-set corresponds to a scenario where there is a partial overlap in impressions' types across partners; and the third data-set represents a situation where an advertiser's target set of impression is equally distributed across various levels. In the rest of the section, we refer to the publisher data set of generated using method A (similarly B and C) as data-set A (similarly B and C).

\section{Performance Metrics.}

We next illustrate the key metrics used to measure the performance of various algorithms. 
Fill Rate: Given priority level $\ell$, the fill-rate for a publisher at level $\ell$ is the fraction of impressions at level $\ell$ that are assigned an advertisement. As we discussed in the introduction, the publisher wants to keep the fill rate as high as possible for high-priority partners. This is the most important metric for measuring the performance of an algorithm.

Under-delivery: It is the total unmet demand summed over all advertisers, expressed as a fraction of total advertiser demand. In order to satisfy contracts, it is important to keep the under-delivery as small as possible.

Over-delivery: We say that an advertiser is over-delivered when he has been assigned more impressions than his demand. We note, no additional revenue is generated by overserving an advertiser. Since a publisher can delegate unassigned impressions at run-time to other advertising markets (such as Google AdExchange, Yahoo! Right Media) to generate additional revenue, it is imperative to keep the overdelivery as low as possible.

We measure the over-delivery as the total number of overdelivered impressions summed over all over-delivered advertisers, expressed as the fraction of total advertisers' demand.

Total Weight of Matching (Demand Capped): We associate the CTR for an impression-advertiser pair as the weight on the edge between them. Thus the total weight of the matching represents the total number of clicks generated for advertisers. Modulo other constraints, the publisher prefers to keep the total weight of the matching as high as possible, as the advertisers' welfare is typically measured in terms for clicks generated for them.

We measure the demand capped weight of the matching; i.e. we only consider the top $N(i)$ edges for advertiser $\mathcal{A}_{i}$ towards the total matching weight.

\section{Algorithms and Their Parameters.}

We perform exhaustive experimental analysis of various algorithms to measure their performance; we next enlist various algorithms and the set of parameters in each algorithm used in the experimental analysis.

DUAL-SCALE: The different values of $\beta$ used in the DUALSCALE algorithm are $\{0,0.1,0.2,0.5,0.9,1.0\}$.

EW-SCALE: We use four different sets of scaling factors for the EW-SCALE algorithm. We illustrate the scaling factor for an impression at level $\ell$ in these sets:

Set 1: The scaling factor is $(1-0.1 *(\ell-1))$ (linear scaling).

Set 2: The scaling factor is $(2-0.1 *(\ell-1))$ (linear scaling).

Set 3: The scaling factor is $0.9^{\ell-1}$ (exponential scaling).

Set 4: The scaling factor is $0.8^{\ell-1}$ (exponential scaling).

Use Both Techniques Simultaneously: We also run experiments where we run both techniques simultaneously on an impression: when an impression at level $\ell$ arrives, we scale its weight according to the edge-weight scaling parameter; we then use the dual-scaling technique to compute the value of the dual for each advertiser.

Baseline: We use the PD-Avg algorithm by [12] as the baseline algorithm. This algorithm ignores impressions' levels, each advertiser's dual is the average weight of impressions assigned to him so far, and each incoming impression is assigned to an advertiser that maximizes the marginal between the edge-weight and his dual.

\subsection{Experiments and Observations}

Experiment 1: Comparison of Various Algorithms.

We first compare the performance of all four algorithms; for the DUAL-SCALE algorithm, we use $\beta=0.5$, and for the EW-SCALE algorithm, we use scaling set 3. Experimental results are given in Figures 5 (fill rates) and 3 (overall delivery metrics). We note salient observations; we begin with the DUAL-SCALE algorithm.

For each publisher and its each data-set, the DUAL-SCALE algorithm has less under-delivery and more over-delivery than the baseline algorithm. Further, for each publisher, its underdelivery decreases from data-set $A$ to data-set $C$; and the opposite is true for over-delivery., For example, in the case of publisher 2, the under-delivery decreases from 21 to 19.5 to 18 (from data-set A to $\mathrm{C}$ ), whereas the over-delivery increases from 12.5 to 14.5 to 16 . We next illustrate the reasons for these observations: let $\mathcal{I}_{j}$ be an incoming impression and its level is $\ell$. Before $\mathcal{I}_{j}$ 's arrival, assume that the DUALSCALE algorithm and the baseline algorithm have assigned the same set of impressions to advertiser $\mathcal{A}_{i}$; let this set be $S_{i}$. As the dual scaling algorithm discounts the weight of impressions in level below $\ell$, we get:

$$
\gamma(i, \ell) \leq \gamma(i, L)
$$

Thus, the marginal gain for assignment to $\mathcal{A}_{i}$ in the dual scaling algorithm $(w(i, j)-\gamma(i, \ell)))$ dominates the same in the baseline algorithm $(w(i, j)-\gamma(i, L))$. Hence, the DUALSCALE algorithm will always assign an impression which would have been assigned by the baseline algorithm (assuming identical earlier assignment to all advertisers). However, its converse is not true: the DUAL-SCALE algorithm can also allocate impressions when the advertiser is fully allocated and the incoming impression is no better (in terms of its weight) compared to the average assignment to the advertiser. Thus the algorithm is prone to over-deliver (and, hence has lower under-delivery) compared to the baseline algorithm. Further, as we go from data-set A to data-set C, the target set of impressions for an advertiser is more evenly distributed across various levels. As a result, we expect this effect to amplify. This results in the observed patterns in under-delivery and over-delivery across data-sets for a single publisher. We also note, as a side-effect of less under-delivery, we see an increase in the number of clicks from data-set A to data-set C (with exception of publisher 2).

We observe an exact opposite pattern for the EW-SCALE algorithm. The EW-SCALE algorithm always has more underdelivery and less over-delivery than the baseline algorithm for each publisher. Further, for each publisher, its underdelivery increases from data-set $A$ to data-set $C$ and the opposite is true for over-delivery. We illustrate the reasons for these observations: the algorithm scales down the edgeweights for lower level impressions (compared to higher level impressions). Hence, even when an advertiser has unmet demand and the average weight of impressions assigned to him is lower than the edge-weight for an incoming impression, the impression may remain unassigned as it may now have negative marginal gain after edge-weight scaling; in comparison, this impression would have qualified for assignment in the baseline algorithm. As a result, we see more under-delivery and less over-delivery in the EW-SCALE algorithm compared to the baseline algorithm. Further, as an advertiser's target set is more evenly distributed, we expect this effect to 


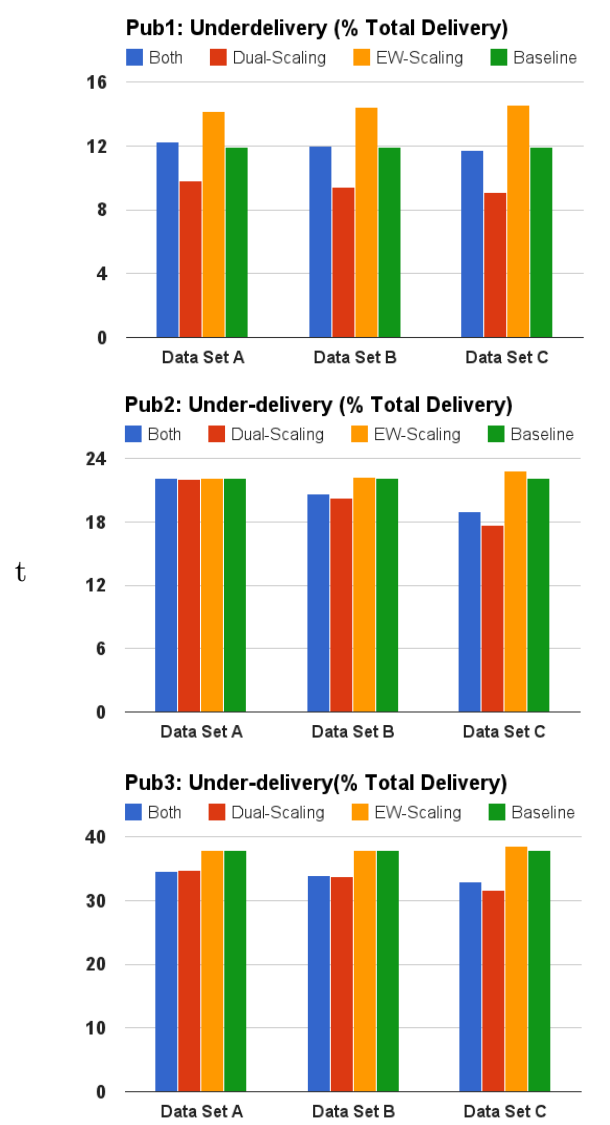

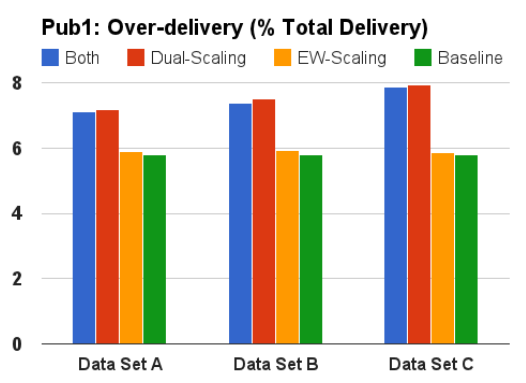

Pub2: Over-delivery (\% Total Delivery)

20

Both Dual-Scaling EW-Scaling Baseline

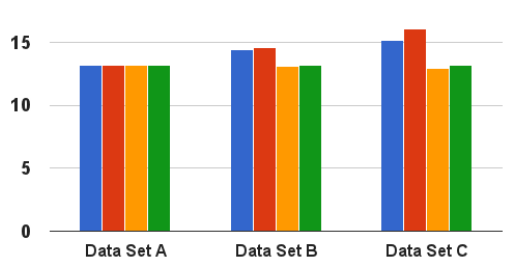

Pub3: Over-delivery(\% Total Delivery)

Both Dual-Scaling EW-Scaling Baseline

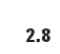

2.8

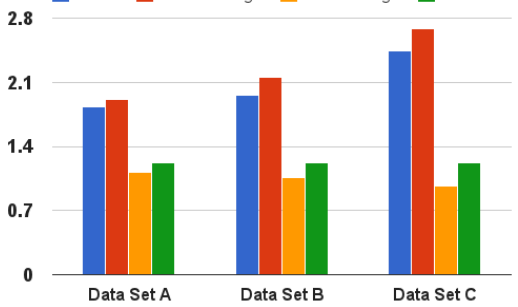

Pub1: Clicks(\% Diff from Baseline)

Both Dual-Scaling EW-Scaling

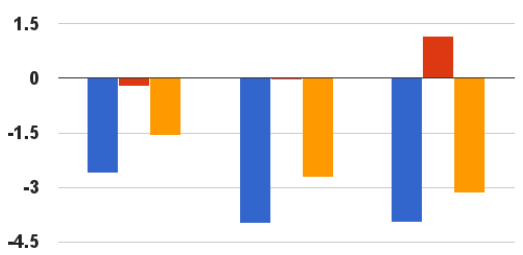

Data Set A Data Set B Data Set C

Pub2: Clicks (\% Diff from Baseline)

Both Dual-Scaling EW-Scaling

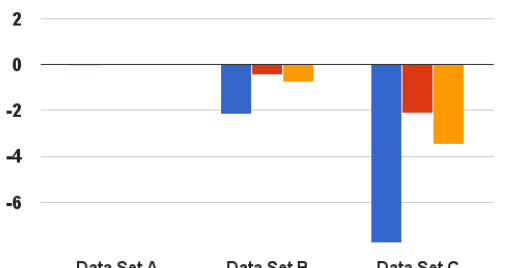

Data Set A Data SetB $\quad$ Data Set C

Pub3: Clicks (\% Diff from Baseline)

Figure 3: Overall Delivery Guarantees for Various Algorithms and Each Publisher: The plots in the $i$ th row are under-delivery, over-delivery and total number of clicks observed in data-sets $A$ through $C$ for publisher $i$. We plot the over-delivery and the under-delivery as a fraction of total delivery; and the number of clicks are represented as percentage difference from the baseline algorithm.
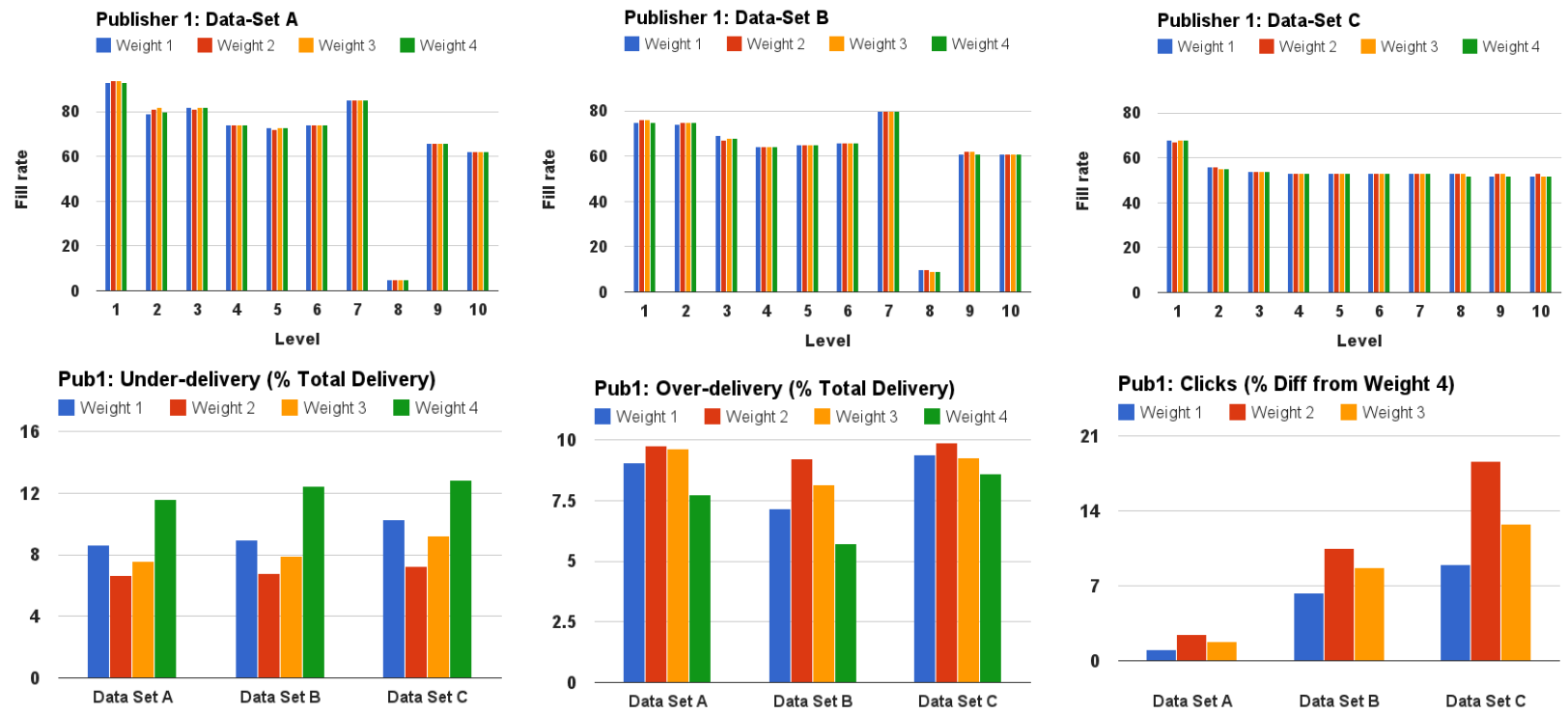

21

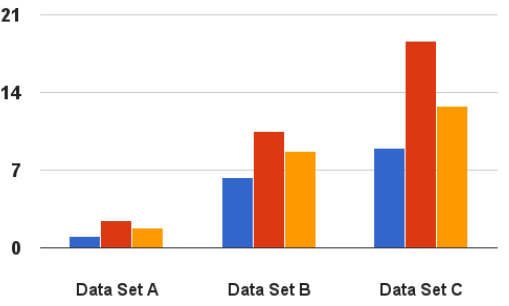

Figure 4: Scaling Factors in EW-SCALE: The plots in the first row are fill-rates for various scaling factors in the EW-SCALE algorithm for one publisher. In the second row, we study their impact on over-all quality metrics, such as under-delivery, over-delivery and number of clicks. 

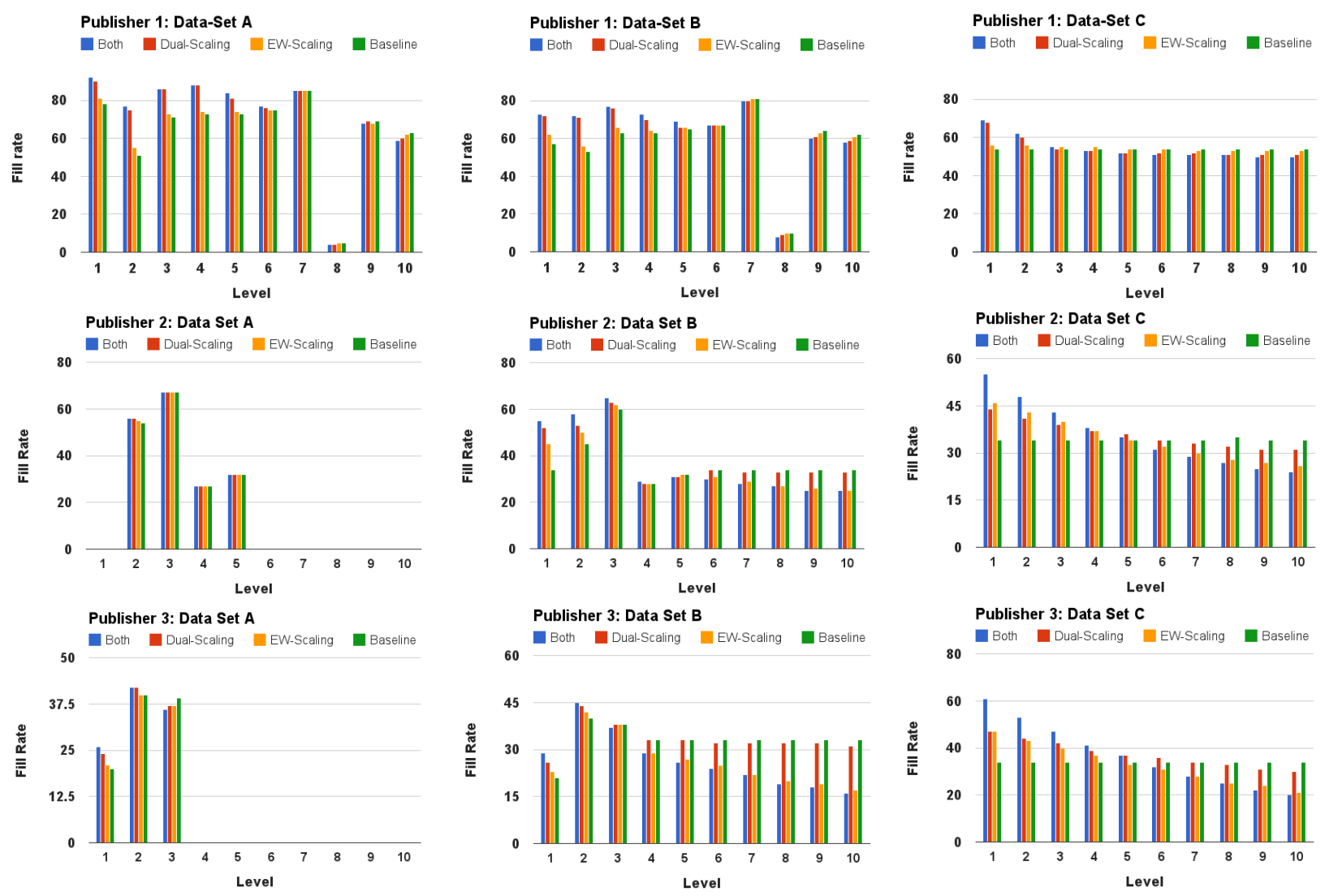

Figure 5: Fill Rates for Various Algorithms for Each Publisher: The plots in the $i$ th row are fill-rates observed in data-sets $A$ through $C$ for publisher $i$. The fill-rates are represented at the percentage of impressions at a level that are assigned ads.
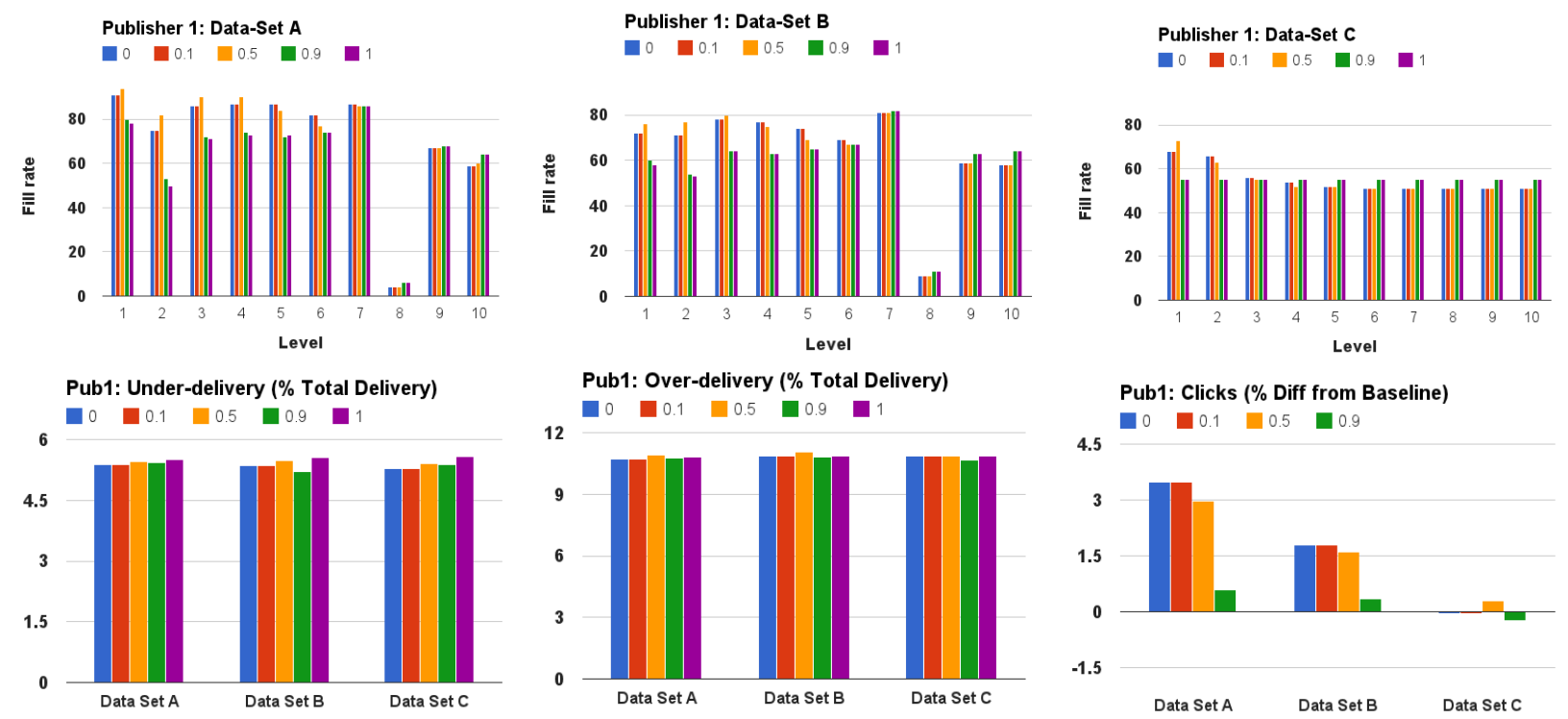

Figure 6: Effect of $\beta$ on DUAL-SCALE: The plots in the first row are fill-rates for various values of $\beta$ for three data-sets generated for a publisher. In the second row, we plot over-all quality metrics, such as under-delivery, over-delivery and number of clicks as a function of $\beta$. 
amplify. As a result, we get the said patterns in the overdelivery and the under-delivery across data sets for any given publisher. Also, as a result of increased under-delivery, the number of clicks reduces from data-set A to data-set $\mathrm{C}$.

We next discuss the observations in fill rates for various algorithms: we see that the fill rates at higher levels are significantly superior for the DUAL-SCALE algorithm compared to the EW-SCALE algorithm (with an exception of publisher 2 for data-set $\mathrm{C}$ where the EW-SCALE algorithm is slightly superior). Further, we see that the fill rates at lower levels for the EW-SCALE algorithm are significantly lower than the DUAL-SCALE algorithm; the reduced delivery at lower levels in the EW-SCALE algorithm is associated with the increased under-delivery of the EW-SCALE algorithm (discussed earlier). In comparison, the reduction in the fill rates at lower levels in the DUAL-SCALE algorithm (compared to the baseline algorithm) is less; and the algorithm is overall more robust.

We also note, using both techniques simultaneously gives a marginal benefit in terms of fill rates at higher levels compared to the DUAL-SCALE algorithm; however the penalty in terms of increased under-delivery far outweighs the gain.

\section{Experiment 2: Scaling Factors in EW-SCALE.}

We next measure the impact of choice of edge-weight scaling factors on the key performance metrics of the EW-SCALE algorithm. Experimental observations for one publisher are given in Figure 4; other publishers yield similar results.

We note, among the four scaling factor sets, the set 4 (approximately) gives the highest bias to higher levels compared to lower levels, followed by set 1 , set 3 and set 2 (in that order). We see that the under-delivery decreases (and the over-delivery increases) in the same order across all data sets for each publisher. This happens as a result of the delivery property of the EW-SCALE algorithm discussed in experiment 1: we expect the said effect to increase with increase in scaling bias, which is consistent with experimental observations.

We also observe that the total weight of matching increases in the order opposite of bias; this is related to the reduction in under-delivery in that order. We note, the experiments do not reveal any significant improvement in terms of fill rates at higher levels for a stronger bias.

\section{Experiment 3: Effect of $\beta$ on DUAL-SCALE.}

We next measure the performance of the DUAL-SCALE algorithm for various values of $\beta$. We discard the real edges weights in this experiment and consider an edge-weight to be 1 , when an impression is in the advertiser's target set, and 0 otherwise. This relates to the scenario where the advertising contracts have significantly higher priority than the clicks generated for the advertisers. Experimental observations for one publisher are given in Figure 6. Note, for $\beta=1$, the algorithm degenerates to baseline.

Salient observations: the under-delivery improves from $\beta=1$ to $\beta=0$; further, for smaller $\beta$, it improves as we go from data-set A to data-set $C$. This happens due to the delivery property of the DUAL-SCALE algorithm discussed in experiment 1. As a result of reduced under-delivery, we also see an increase in the number of clicks as the value of $\beta$ is reduced. In terms of fill rates, we observe that the fill rates at higher levels reduce as we increase $\beta$ from 0.5 to 1.0 . $\mathrm{Cu}$ riously, we get better fill rates for $\beta=0.5$ over $\beta=0.0$. This happens as these algorithms are not implemented in isolation; they are implemented in a framework that simulates all complexities of a real system that handles live traffic and optimizes from various different perspectives.

\section{REFERENCES}

[1] emarketer. http://www.emarketer.com/Article/ Digital-Account-One-Five-Ad-Dollars/1009592, 2013.

[2] Flurry Five-Year Report: It's an App World. The Web Just Lives in It. http://blog.flurry.com/bid/95723/, 2013.

[3] S. Agrawal, Z. Wang, and Y. Ye. A dynamic near-optimal algorithm for online linear programming. Working paper at http://www.stanford.edu/ yyye/.

[4] S. Balseiro, J. Feldman, V. S. Mirrokni, and S. Muthukrishnan. Yield optimization of display advertising with ad exchange. In $A C M-E C, 2011$.

[5] S. R. Balseiro, O. Besbes, and G. Y. Weintraub. Yield optimization of display advertising with ad exchange. In $A C M-E C, 2013$.

[6] A. Bhalgat, J. Feldman, and V. Mirrokni. Online allocation of display ads with smooth delivery. In $K D D, 2012$.

[7] V. Bharadwaj, P. Chen, W. Ma, C. Nagarajan, J. Tomlin, S. Vassilvitskii, E. Vee, and J. Yang. Shale: an efficient algorithm for allocation of guaranteed display advertising. In $K D D, 2012$.

[8] N. Buchbinder, K. Jain, and J. Naor. Online Primal-Dual Algorithms for Maximizing Ad-Auctions Revenue. In Proc. ESA, page 253. Springer, 2007.

[9] D. F. Ciocan and V. Farias. Model predictive control for dynamic resource allocation. In Mathematics of Operations Research, 2012.

[10] N. Devanur and T. Hayes. The adwords problem: Online keyword matching with budgeted bidders under random permutations. In ACM EC, 2009.

[11] J. Feldman, M. Henzinger, N. Korula, V. Mirrokni, and C. Stein. Online stochastic packing applied to display ad allocation. In ESA, 2010.

[12] J. Feldman, N. Korula, V. Mirrokni, S. Muthukrishnan, and M. Pal. Online ad assignment with free disposal. In WINE, 2009.

[13] A. Ghosh, P. McAfee, K. Papineni, and S. Vassilvitskii. Bidding for representative allocations for display advertising. In WINE, pages 208-219, 2009.

[14] K. Lang, J. Delgado, D. Jiang, B. Ghosh, S. Das, A. Gajewar, S. Jagadish, A. Seshan, C. Botev, M. Bindeberger-Ortega, S. Nagaraj, and R. Stata. Efficient online ad serving in a display advertising exchange. In WSDM, 2011.

[15] A. Mehta, A. Saberi, U. Vazirani, and V. Vazirani. Adwords and generalized online matching. In FOCS, 2005.

[16] B. Tan and R. Srikant. Online advertisement, optimization and stochastic networks. CoRR, abs/1009.0870, 2010.

[17] E. Vee, S. Vassilvitskii, and J. Shanmugasundaram. Optimal online assignment with forecasts. In $A C M$ $E C, 2010$. 